\title{
Practical augmented reality (AR) technology and its applications
}

C) Springer Science+Business Media, LLC, part of Springer Nature 2020

Multimedia Tools and Applications gratefully acknowledges the editorial work of the scholars listed below on the special issue entitled "Practical Augmented Reality (AR) Technology and its Applications" (SI 1132 T).

Of 44 papers submitted to this issue, 25 were eventually accepted after a stringent peer review process.

\section{Corresponding Guest Editor}

Jin-Ho Choi

Korea Institute of Information Technology \& Innovation, Seoul, South Korea

Email: mike@kiiti.org

\section{Guest Editors}

\section{Su Ruidan}

Shanghai Advanced Research Institute, Chinese Academy of Sciences, China

Email: surd@sari.ac.cn

\section{Shuai Liu}

Inner Mongolia University, China

Email: cs_liushuai@imu.edu.cn

\section{Hyun-Jong Cha}

Sunmoon University, South Korea

Email: hyunjongcha@naver.com

Publisher's note Springer Nature remains neutral with regard to jurisdictional claims in published maps and institutional affiliations. 